Penteado, C., Cervi, E. y Campos-Domínguez, E. (2021): Polarización en Twitter. El caso de Brasil a través de cinco conflictos políticos. Cultura, Lenguaje y Representación, Vol. XXVI, 127-147

ISSN $1697-7750 \cdot$ E-ISSN 2340-4981

DOI: http://dx.doi.org/10.6035/clr.5837

\title{
Polarización en Twitter. El caso de Brasil a través de cinco conflictos políticos ${ }^{1}$
}

Ideological polarisation on Twitter. The case of Brazil throught five political conflicts

Claudio LUIS DE CAMARGO PENTEADO
UNIVERSIDAD FEDERAL DO ABC (BRASIL)
HTTPS://ORCID.ORG/0000-0002-8279-3643

EMERSON UEIZZI CERVI

UNIVERSIDAD FEDERAL DO PARANÁ (BRASIL)

HTTPS://ORCID.ORG/0000-0001-8073-014X

EVA CAMPOS-DOMÍNGUEZ

UNIVERSIDAD DE VALLADOLID (ESPAÑA)

HTTPS://ORCID.ORG/0000-0002-8970-7947

Artículo recibido el / Article received: 2021-03-31

Artículo aceptado el / Article accepted: 2021-09-20

RESUMEN: Esta investigación se centra en el análisis de las transformaciones en la esfera pública digital. Tiene por objetivo estudiar los perfiles de Twitter en la polarización de conflictos políticos discursivos. A través del análisis de cinco conflictos diferentes en Brasil, registrados durante el año 2020, este trabajo analiza las 500 cuentas que registraron un mayor número de retuits dentro de una muestra de 447.762 mensajes recogidos mediante hashtags, que sumaron 334.588 retuits. Aplicando un análisis de residuos estandarizados, mediante lenguaje $\mathrm{R}$, se plantea como objetivos identificar si efectivamente existe polarización ideológica en el debate público en Twitter e identificar el tipo de perfil que genera, en su caso, esa polarización en Twitter. Aunque los datos atañen únicamente a un estudio de caso concreto en Brasil, los resultados aportan valor para un análisis más amplio, dado que el contexto político y social

\footnotetext{
${ }^{1}$ Projeto Observatório de Conflitos na Intenet (Cooperação Científica e Tecnológica entre FAPESP, MCTI, MC e CGI - Brasil).
} 
brasileño plantea elevados niveles de confrontación y polarización política con proyección internacional. Los resultados permiten señalar que la esfera pública digital es dinámica, con el predominio de la personalización y customización del debate público, incrementado con la entrada de nuevos actores o fuentes en la discusión política y la pérdida de espacio como fuente de información en el debate público por parte de actores clásicos como los medios de comunicación.

Palabras clave: Comunicación política, esfera pública digital, Twitter, Brasil

ABSTRACT: This research focuses on transformations in the digital public sphere. Its objective is to analyze Twitter profiles in the polarization of discursive political conflicts. Through the analysis of five different conflicts in Brazil, registered during 2020, this work studies the 500 accounts that registered the highest number of retweets within a sample of 447,762 messages collected through hashtags, which totaled 334,588 retweets. Applying a standardized residual analysis, using $\mathrm{R}$ language, the objectives are to identify if there is indeed ideological polarization in the public debate on Twitter and to identify the type of profile that generates, if applicable, that polarization on Twitter. Although the data refer only to a specific case study in Brazil, the results provide value for a broader analysis, given that the Brazilian political and social context raises high levels of confrontation and political polarization with international projection. The results allow us to point out that the digital public sphere is dynamic, the predominance of personalization and customization of the public debate, increased with the entry of new actors or sources in the political discussion and the loss of space as a source of information in the public debate by part of classical actors such as the media.

Key words: Political communication, digital public sphere, Twitter, Brazil

\section{INTRODUCCIÓN}

Las revueltas que, tras la primavera árabe, se sucedieron en diferentes países del mundo, planteaban que Internet era capaz de neutralizar los espacios comunicativos de las élites políticas y mediáticas tradicionales con la participación de la ciudadanía (Castells, 2012; Gerbaudo, 2012). En Brasil, desde las movilizaciones celebradas en junio de 2013 que tomaron las calles de diferentes ciudades del país, las redes sociales han ido ganando relevancia e influyendo en procesos políticos, como fue en las manifestaciones a favor y en contra del impeachment (proceso de destitución) de la presidenta Dilma Roussef en 2015 y 2016 (Carvalho et al., 2016) o, entre otros, en las elecciones presidenciales de 2018 (Soares et al., 2018; Oliveira et al., 2020).

Como también ocurre en otros países, las redes sociales se han convertido en un espacio central para el debate político contemporáneo en: a) la movilización social de grandes protestas, como la Primavera Árabe, el 15M en España, Occupy Wall Street, entre otras (Castells, 2015); y más recientemente, el movimiento \#Blacklivesmatter (Freelon et al, 2018) y \#MeToo (Orgad y Gill, 2019); b) la formación y elaboración de identidades políticas (Gerbaudo, 2012); c) las campañas electorales digitales (StromerGalley, 2019; Strömbäck y Luengo, 2008); d) la emergencia del populismo digital 
(Luengo y Fernández-García, 2019; Gerbaudo, 2018); o e) la expresión de antagonismos políticos manifestados por medio de la polarización del debate público entre posicionamientos ideológicos diferentes (Kamienski et al., 2021).

El aumento del número de personas conectadas a Internet, principalmente por el auge de las aplicaciones móviles y por la popularización de redes sociales, ha llevado a la formación de un complejo sistema híbrido de medios (Chadwick, 2017), asociado a un proceso de plataformización de la sociedad (van Dijck, Poell y de Waal, 2018) que avanza hacia las «sociedades dataficadas» (van Dijck, 2014). Todo ello, manifiesta una serie de desafíos para comprender el funcionamiento de una esfera pública digital y pone en agenda la discusión del propio concepto de esfera y opinión públicas (De Blasio et al., 2020).

La visión de la esfera pública digital se aleja, para algunos autores, de un espacio de deliberación habermasiano de intercambio y producción de raciones y promueve que cada vez existan menos experiencias sociales compartidas por toda la ciudadanía y la comunidad política: los medios tradicionales ya no producen de forma eficiente ese pegamento social que mantiene a las sociedades plurales más o menos unidas por medio de la exposición a los mismos mensajes sobre los asuntos de interés general y a la diversidad (Wolton, 2000). Ahora tenemos dietas mediáticas personalizadas, a medida, exposición selectiva, polarización (Bimber y Gil de Zúñiga, 2020), que generan nuevas percepciones ciudadanías sobre la recepción de la información (Gualda y Rúas, 2019).

En la nueva configuración de la esfera pública, en la que las plataformas de redes sociales se convierten en una de las principales formas de socialización y acceso a la información (Dufva y Dufva, 2019), los usuarios pueden participar en la discusión sobre diferentes temas, contribuir con nuevas fuentes de información y expresar sus posicionamientos políticos, haciendo el discurso más plural (Serrano-Contreras et al. 2020) y transformando el entorno comunicativo en todos los procesos informativos (Casero-Ripollés, 2018).

El aumento de esta pluralidad también motiva la emergencia de conflictos entre los usuarios, que pueden mostrar no solo visiones del mundo diferentes, sino también las expresiones de polarización ideológica (Souza et al., 2017), disputas por los enfoques de acontecimientos públicos en la lucha por la hegemonía (Laclau y Mouffe, 2014) y la influencia de la opinión pública. En este sentido, la comunicación digital ha promovido una nueva mediatización vinculada a la adecuación de los mensajes políticos al discurso, estrategias y lógicas de los medios, pero al mismo tiempo mantienen la dependencia a los medios de comunicación (Casero-Ripollés et al, 2016; López-García, 2017).

El aumento de esta pluralidad también motiva la emergencia de conflictos entre los usuarios, que pueden mostrar no solo visiones del mundo diferentes, sino también las expresiones de polarización ideológica (Souza et al., 2017), disputas por los enfoques de acontecimientos públicos en la lucha por la hegemonía (Laclau y Mouffe, 2014) y la influencia de la opinión pública.

Con el objetivo de ahondar en las transformaciones en la esfera pública digital, este artículo presenta un estudio sobre el papel de los perfiles en la polarización de conflictos políticos discursivos en Twitter. A través del análisis de cinco conflictos diferentes en Brasil, registrados durante el año 2020, este trabajo se plantea como objetivos (1) identificar si existe polarización ideológica en la muestra seleccionada y (2) identificar el tipo de perfil que genera, en su caso, esa polarización en Twitter.

Desde la campaña electoral de 2014, Brasil ha venido atravesando un proceso de radicalización de la polarización política. Este proceso se ha manifestado en un embate entre usuarios en redes sociales en defensa de candidatos de campos ideológicos opuestos (Chaia y Brugnago, 2014). La intensificación de los conflictos discursivos en redes 
sociales durante las manifestaciones del impeachment de Dilma Rousseff (2015-2016) ha dividido a los brasileños entre los partidarios de la destitución de la presidenta, asociados al campo ideológico de la derecha, y otros usuarios, alineados al campo ideológico progresista, que denunciaban la existencia de un golpe de Estado en el país con el apoyo de los grupos mediáticos (Carvalho et al. 2016). En las elecciones de 2018, Jair Bolsonaro (el presidente electo) desarrolló su estrategia de campaña en Twitter basada en la construcción de la polarización antagónica con su principal contrincante, el candidato de izquierdas Fernando Haddad del Partido dos Trabalhadores (PT) (Partido de los Trabajadores) (Penteado y Chaves, 2021).

Para investigar los conflictos online, el grupo de investigación en el que se enmarca esta investigación desarrolla una serie de estudios sobre diferentes pugnas que emergen en la Twitter-esfera brasileña. En otros estudios, se ha podido observar la existencia de perfiles polarizadores (Kamienski et al., 2021), la disputa ideológica entre narrativas para combatir el coronavirus (de França et al., 2021) y la disensión dentro del campo ideológico de la propia derecha (entre partidarios del presidente Bolsonaro y los defensores de una agenda liberal) (Santos et al, 2020).

Para este estudio, seleccionamos cinco conflictos con diferentes temáticas (salud, economía, gobierno, protestas y educación) que tuvieron lugar durante el año 2020 en Brasil para estudiar el papel de los perfiles responsables de producir las principales narrativas en relación con los eventos seleccionados.

\section{REDES SOCIALES Y ESFERA PÚBLICA DIGITAL}

Un elemento central para comprender las transformaciones de la esfera pública digital es el estudio de las interacciones entre los perfiles de usuarios en redes sociales y la formación de opinión pública. Las plataformas digitales asumen una centralidad como espacio de debate público. Estructurada en una arquitectura de red distribuida (Benkler, 2006), la esfera pública digital fomenta un debate más plural, abierto para todos los usuarios conectados en plataformas digitales, sin la mediación de los tradicionales intermediarios y gatekeepers. Esa pluralidad permite la ampliación de la participación ciudadana, la diversidad de fuentes de información y la entrada de nuevos actores en el debate público. Por otro lado, el flujo de informaciones es mediado por los algoritmos de las plataformas que influyen en la circulación de los datos y pueden promover la formación de burbujas de filtros (Pariser, 2011; Bruns, 2019) y cámaras de eco, limitando la interacción para personas que piensan de la misma forma y alimentando la fragmentación de la sociedad (Sunstein, 2018), así como también crea un espacio para la difusión de la desinformación y dispositivo de modulación algorítmica (Rouvroy et al., 2013), todo ello demuestra su carácter ambivalente (De Blasio et al., 2020).

En este contexto ambivalente, surgen nuevos modelos de acción y activismo político, como los partidos plataformas o digitales (De Blasio y Viviani, 2020) y los líderes populistas (Gerbaudo, 2018) que se articulan, también, a través de redes sociales. Los tradicionales actores se han adaptado para tener presencia en redes sociales (Barberá y Zeitzoff, 2018). Así es como también emergen nuevos actores políticos y movimientos sociales en la disputa por la opinión pública y en los procesos políticos, transformando las redes sociales en importantes herramientas y espacios para la acción política contemporánea (de Camargo Penteado y Cruz Junior, 2019).

A pesar de que cada plataforma de redes sociales tiene su especificidad, esta comunicación se caracteriza por un proceso de personalización y cambios en la participación política (Bennet, 2012) en los que la interacción se articula en torno a unos pocos perfiles de usuarios. El flujo de la información es controlado por la acción de los 
usuarios y por los algoritmos de las plataformas, en un proceso de customización de los perfiles. Esta customización influye en el consumo de información y en el comportamiento de los usuarios. Un importante factor en este proceso es la formulación de una identidad en la estos y los algoritmos seleccionan seguidores y las fuentes de información de acuerdo con sus preferencias y afinidades.

En la literatura científica no hay consenso sobre los efectos del proceso de customización en el consumo de información en el debate público de las redes sociales. De un lado, los autores apuntan que una de sus consecuencias es la formación de cámaras de eco, en las que los perfiles limitan los contenidos que previamente coinciden, evitando dialogar con otras fuentes de información, llevando a un proceso de fragmentación social (Sunstein, 2018). Por otro lado, otros autores aportan datos que cuestionan este argumento y señalan que los investigadores deberían buscar nuevas preguntas de investigación para la comprensión del debate en las plataformas digitales (Bruns, 2019).

Ambas perspectivas han orientado la realización de estudios sobre la conversación pública en redes sociales. Un tema importante en la agenda académica sobre el funcionamiento de la esfera pública digital es la emergencia de conflictos entre los perfiles de usuarios, llevando a una polarización entre posicionamientos políticos ideológicos (Liu y Weber, 2014). La polarización entre los usuarios puede reflejar semejanzas de debates políticos electorales entre los partidarios de candidatos en disputas electorales (Primario et al., 2017), ampliando y dinamizando la participación política con la entrada de nuevos agentes y fuentes de información, así como también puede intensificar conflictos con la difusión de desinformación, poniendo en riesgo el funcionamiento de la democracia (Tucker et al., 2018).

La preocupación por la polarización y por la desinformación también implica un debate sobre el funcionamiento de la esfera pública, no necesariamente más controlada por los gatekeepers y los medios tradicionales. En relación con el concepto de esfera pública en red (networked public sphere) Benkler et al. (2013) argumentan que la apertura para la participación de nuevos actores (como pueden ser ONG, activistas, sociedad civil, etc.) en el debate público posibilita un aumento de la «conciencia pública» a partir de las interacciones de los usuarios conectados, contribuyendo en la acción política afirmativa.

Por otro lado, Bennett y Pfetsch (2018) señalan que las transformaciones del sistema mediático, en lugar de crear un espacio plural y vibrante para la participación democrática, han producido una esfera pública interrumpida, que está desconectando la ciudadanía de las instituciones públicas y de los medios de comunicación, y amplía la división social. Esta nueva configuración plantea desafíos para la comunicación política que requiere repensar conceptos para la comprensión y la medición de las influencias de las redes sociales sobre los procesos y debates políticos.

Las interacciones de las redes sociales también modifican el proceso de custodia de las informaciones, antes limitadas a los profesionales de la información: periodistas y medios de comunicación comparten la función de producir contenidos, distribuirlos y seleccionar las informaciones que van a ser compartidas con sus seguidores (Bruns, 2019).

En una estructura en la que las relaciones (flujos de información) son establecidas por medio de las redes de seguidores, los diferentes perfiles de usuarios difunden informaciones entre sus contactos que terminan formando parte del debate público, produciendo contenidos, emitiendo opiniones, divulgando mensajes, criticando a otros usuarios; así, emerge el desafío para el campo de la comunicación política de identificar cuáles son esos perfiles, cuáles son sus características, si tienen un alineamiento ideológico y cuáles son los temas que generan una mayor polarización en el debate público online. 
Con el objetivo de estudiar las transformaciones de la esfera pública digital, caracteriza por la polarización entre perfiles de usuarios de medios sociales en la discusión de temas de interés público, como: salud, economía, gobierno, manifestaciones y educación, este artículo pretende responder a las siguientes preguntas de investigación:

RQ1. ¿Existe un proceso de polarización ideológica entre los perfiles en la Twitter-esfera brasileña?

RQ2. ¿Cuáles son los principales tipos de perfiles en el debate público brasileño en Twitter en los casos de conflicto seleccionados?

\section{METODOLOGÍA}

Para llevar a cabo este estudio, se analizan las conservaciones en Twitter a partir de cinco asuntos que causaron conflicto político en Brasil, todos los casos analizados tuvieron lugar en el año 2020, en medio de la pandemia de coronavirus. Como criterio de selección, fueron elegidos temas del área de salud pública (asociado a medidas para combatir la Covid-19), economía, gobierno federal, movilización para protestas y educación. Para ello, tomamos en consideración estudios previos como Chaves-Montero et al (2021) para centrarnos en la red social Twitter como la principal herramienta de comunicación digital durante el periodo de confinamiento.

Tabla 1. Casos de estudio seleccionados: contexto y datos generales

\begin{tabular}{|c|c|c|c|}
\hline & $\begin{array}{l}\text { Tema de } \\
\text { conflicto }\end{array}$ & Descripción y contexto & Datos de la recogida \\
\hline 1 & $\begin{array}{l}\text { Salud pública / } \\
\text { pandemia }\end{array}$ & $\begin{array}{l}\text { Tuvo lugar a partir del } 15 / 03 / 2020 \text {, debido al } \\
\text { crecimiento de casos de personas infectadas por } \\
\text { COVID- } 19 \text { en Brasil. La falta de un plan federal } \\
\text { para afrontar la pandemia provocó que } \\
\text { gobernadores y alcaldes de diferentes estados y } \\
\text { municipios adoptaran diferentes medidas de } \\
\text { lucha contra la pandemia, generando un debate } \\
\text { intenso en Twitter sobre la suspensión de las } \\
\text { clases, el cierre de fronteras y el confinamiento } \\
\text { en las ciudades. }\end{array}$ & $\begin{array}{l}\text { Fecha/hora del } 1^{\circ} \text { tweet } \\
\text { procesado } \text { (Greenwich): } \\
\text { 15/03/2020 - 00:12 } \\
\text { Fecha/hora del último tweet } \\
\text { (Greenwich): } 23-03-2020 \text { - } \\
\text { 19:28 } \\
\text { Total tweets: } 232.481 \\
\text { Total retweets: } 166.322 \text { - } \\
0,71 \% \\
\text { Total respuestas: } 5.378 \text { - } \\
0,02 \%\end{array}$ \\
\hline 2 & $\begin{array}{l}\text { Economía/ } \\
\text { pandemia }\end{array}$ & $\begin{array}{l}\text { Tuvo lugar a partir del día } 07 / 05 / 2020 \text {. A partir } \\
\text { de las medidas adoptadas por gobernadores y } \\
\text { alcaldes para restringir las actividades } \\
\text { comerciales, un grupo de empresarios se reunió } \\
\text { con el presidente Bolsonaro para exigir medidas } \\
\text { de protección a las empresas. Un comunicado } \\
\text { de uno de los empresarios señalaba que si el } \\
\text { gobierno federal no hacía nada «Habrá muertes } \\
\text { del CNPJ», refiriéndose al cierre de empresas } \\
\text { por medidas de aislamiento social. La } \\
\text { declaración provocó una reacción inmediata en } \\
\text { la Twitter-esfera brasileña, entre los contrarios } \\
\text { al cierre de las empresas y los que abogaban por } \\
\text { «salvar vidas» como la prioridad de las } \\
\text { autoridades públicas. }\end{array}$ & 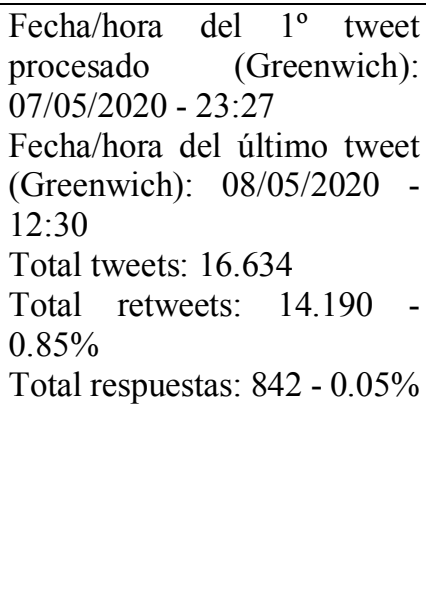 \\
\hline 3 & $\begin{array}{l}\text { Gobierno } \\
\text { federal/ política }\end{array}$ & $\begin{array}{l}\text { Para estudiar el debate sobre las polémicas en } \\
\text { torno al gobierno del presidente Jair Bolsonaro, } \\
\text { se selecciona el conflicto en Twitter relacionada } \\
\text { con una declaración del General Heleno, } \\
\text { ministro de Seguridad Institucional, señalando }\end{array}$ & $\begin{array}{l}\text { Fecha/hora del } 1^{\circ} \text { tweet } \\
\text { procesado } \\
\text { 22/05/2020 }\end{array}$ \\
\hline
\end{tabular}




\begin{tabular}{|c|c|c|c|}
\hline & & $\begin{array}{l}\text { que una eventual incautación del teléfono móvil } \\
\text { del presidente, para una investigación policial, } \\
\text { podría tener «consecuencias imprevisibles». } \\
\text { Este hecho tuvo lugar el } 22 / 05 / 2020 \text {. Es preciso } \\
\text { enmarcar estas declaraciones en el contexto de } \\
\text { que, en Brasil, hay una gran preocupación } \\
\text { porque Bolsonaro, ex militar y defensor del } \\
\text { régimen militar brasileño (1934-1985), pueda } \\
\text { usar su cargo para implantar un nuevo golpe } \\
\text { militar. La política de los partidos de oposición } \\
\text { generó una rápida repercusión en Twitter de que } \\
\text { el ministro general estaría haciendo una } \\
\text { amenaza a la democracia brasileña. }\end{array}$ & $\begin{array}{l}\text { Fecha/hora del último tweet } \\
\text { (Greenwich): } 23 / 05 / 2020- \\
12: 21 \\
\text { Total tweets: } 42.120 \\
\text { Total retweets: } 35.061- \\
0.83 \% \\
\text { Total replies: } 3.025-0.07 \%\end{array}$ \\
\hline 4 & Manifestaciones & $\begin{array}{l}\text { Esta muestra de datos recogida se relaciona con } \\
\text { el debate em Twitter sobre las manifestaciones } \\
\text { de grupos de aficionados al fútbol que tuvieron } \\
\text { lugar el } 03 / 06 / 2020 \text {. En medio de la pandemia, } \\
\text { las manifestaciones en la calle promovidas por } \\
\text { segmentos de hinchas del fútbol fueron } \\
\text { asociadas a una respuesta a las protestas de } \\
\text { seguidores del presidente Bolsonaro, en favor } \\
\text { de una intervención militar en el país. Las } \\
\text { manifestaciones originaron un conflicto } \\
\text { implicando la lucha discursiva entre los } \\
\text { términos de fascistas y antifascistas en las redes } \\
\text { sociales. }\end{array}$ & $\begin{array}{l}\text { Fecha/hora del } 1^{\circ} \text { tweet } \\
\text { procesado } \\
\text { (Greenwich):03/06/2020 - } \\
\text { 16:16 } \\
\text { Fecha/hora del último tweet } \\
\text { (Greenwich): 04/06/2020 - } \\
\text { 20:38 } \\
\text { Total tweets: } 90.968 \\
\text { Total retweets: } 65.324 \quad- \\
0.71 \% \\
\text { Total replies: } 12.052-0.13 \%\end{array}$ \\
\hline 5 & Educ & $\begin{array}{l}\text { El último conflicto seleccionado se relaciona } \\
\text { con el decreto de la Medida Provisória } \\
979 / 2020,10 / 06 / 2020 \text {, que autorizó el ministro } \\
\text { de Educación brasileño, Abraham Weintraub, y } \\
\text { que permitía escoger a los rectores temporales } \\
\text { de instituciones universitarias federales de } \\
\text { educación durante la pandemia. Esta medida iba } \\
\text { en contra de la autonomía universitaria } \\
\text { existente. El decreto motivo una rápida reacción } \\
\text { en Twitter, que usaron la red para atacar o para } \\
\text { defender al ministro de Educación y al } \\
\text { Gobierno de Bolsonaro. }\end{array}$ & $\begin{array}{l}\text { Fecha/hora del } 1^{\circ} \text { tweet } \\
\text { procesado: } \\
\text { (Greenwich): } 10 / 06 / 2020- \\
16: 35 \\
\text { Fecha/hora del último tweet } \\
\text { (Greenwich): } 11 / 06 / 2020- \\
\text { 16:30 } \\
\text { Total tweets: } 65.559 \\
\text { Total retweets: } 53.691 \quad- \\
0.81 \% \text { r } \\
\text { Total respuestas: } 2.634 \quad- \\
0.04 \%\end{array}$ \\
\hline
\end{tabular}

El análisis de los conflictos se realizó en dos etapas: en una primera, se identificaron los principales flujos movilizados discursivamente en los casos estudiados $\mathrm{y}$, en una segunda etapa, se realizó un análisis residual, conforme se describe a continuación.

La primera etapa consistió en la identificación de las formaciones discursivas; es decir, los flujos movilizados mediante elementos discursivos (tweets), en el proceso de interacción dialógica online (Kent y Taylor, 2002). Con el análisis de los mensajes con mayor engagement, se identificaron las principales formaciones discursivas utilizadas por los agentes implicados en los conflictos seleccionados, direccionando la disputa discursiva en torno a los efectos de significado entre los sujetos implicados sobre determinada realidad o acontecimiento (Narvaz et al, 2006).

El análisis residual fue realizado a partir de los mensajes recogidos en Twitter en torno a los hashtags más usados en cada uno de estos conflictos, seleccionamos las cien cuentas con los mensajes más retuiteados y compartidos en cada conflicto señalado. Consideramos, así, esas cien cuentas como el grupo más influyente en cada uno de los cinco asuntos descritos en la tabla anterior (Tabla 1). Para cumplir los objetivos del 
trabajo, clasificamos estas cuentas en dos dimensiones, conforme a las categorías y tipologías planteadas por Kamienski et al (2021):

Tabla 2. Tipología de perfiles según origen y descripción ideológica

\begin{tabular}{|c|c|}
\hline Clasificación & Descripción \\
\hline \multicolumn{2}{|c|}{ Perfiles institucionales y colectivos } \\
\hline $\begin{array}{l}\text { Medio } \\
\text { tradicional }\end{array}$ & Perfiles asociados a la prensa tradicional \\
\hline $\begin{array}{l}\text { Medios } \\
\text { alternativos }\end{array}$ & $\begin{array}{l}\text { Perfiles asociados a la producción de información alternativa a la prensa } \\
\text { tradicional; algunos de estos perfiles tienen una clara posición política } \\
\text { expresada en su contenido }\end{array}$ \\
\hline Institución & Perfiles de instituciones gubernamentales públicas \\
\hline Grupo político & $\begin{array}{l}\text { Perfiles asociados a grupos y colectivos que actúan en defensa de sus causas } \\
\text { políticas (ej: defensa del conservadurismo, uso de armas, derechos } \\
\text { humanos, movimientos de vivienda, etc.) }\end{array}$ \\
\hline \multicolumn{2}{|c|}{ Perfiles personales (individual) } \\
\hline Celebridades & $\begin{array}{l}\text { Perfiles asociados con personajes famosos, que involucran actores de } \\
\text { televisión, cantantes y otras personalidades muy visibles. }\end{array}$ \\
\hline Influencers & Perfiles con gran número de seguidores (más de 1 millón) \\
\hline Periodista & Perfiles de periodistas \\
\hline Político & Perfiles asociados a políticos (con o sin cargo político) \\
\hline Tuitero & Perfiles de personas con más de 10.000 seguidores \\
\hline Usuario común & Perfiles de personas comunes con menos de 10.000 seguidores \\
\hline \multicolumn{2}{|c|}{ Posición ideológica } \\
\hline Clasificación & Descripción \\
\hline Derecha & $\begin{array}{l}\text { Perfiles alineados a posición ideológica derecha, que involucran a partidarios } \\
\text { del presidente Bolsonaro (con agendas conservadoras y autoritarias) y } \\
\text { defensores de la agenda de la economía liberal; }\end{array}$ \\
\hline Izquierda & $\begin{array}{l}\text { Perfiles alineados a la izquierda, que involucran a políticos de oposición, } \\
\text { defensores de agendas asociadas a derechos humanos, medio ambiente y } \\
\text { políticas sociales; }\end{array}$ \\
\hline Indefinidos & $\begin{array}{l}\text { Perfiles asociados a empresas de comunicación tradicionales, sin una } \\
\text { posición política clara y otros perfiles que no se pueden identificar con una } \\
\text { posición clara. }\end{array}$ \\
\hline
\end{tabular}


Tal y como muestra la tabla anterior (Tabla 2), la primera de estas dos dimensiones se considera atendiendo al origen de la cuenta, considerando para ello «medios alternativos», «celebridades», «usuario común», «influencers», «institución», «periodista», «grupo político», «político», «medio tradicional»o «tuitero» cuando hay un número intermedio de seguidores que lo diferencia de un usuario común, pero no se transforma en celebridad. Para los objetivos del análisis aquí propuesto, la dimensión de los tipos de perfiles fue agregada en «cuentas personales», cuando pertenecen a personas físicas, que son: celebridades, periodista, político, tuitero y usuario común., y en «cuentas institucionales» que reúne medios alternativos, tradicionales, grupos políticos y otras instituciones.

La segunda de las dimensiones se estima en relación con el posicionamiento ideológico explícito en la descripción de la cuenta o en mensajes expresos a través de cuentas retuiteadas. Este posicionamiento puede ser: «Derecha» - generalmente vinculados con partidarios y defensores de la agenda conservadora o políticas del Gobierno de Jair Bolsonaro-, «Izquierda» —identificado con perfiles de la oposición brasileña— o también «Indefinido» — cuando no hay manifestación explícita de la alineación a uno de los dos polos anteriores-.

Para el análisis de los perfiles, relacionados con los temas de los conflictos, utilizamos la técnica de análisis de residuos estandarizados para identificar cómo se distribuyeron las categorías de cada dimensión en los temas descritos. En principio, si los residuos eran bajos, las categorías se distribuían aleatoriamente entre los temas; es decir: no había concentración de la presencia de la categoría (Pestana y Gageiro, 2014). Si los residuos estaban distantes a cero, indicaba que los usuarios con determinadas características se concentraban en algunos conflictos. Para verificar el grado de asociación general entre las variables se utilizó el coeficiente V de Cramer.

\section{RESULTADOS}

El análisis de las formaciones discursivas articuladas dialógicamente en los conflictos estudiados se presenta en la Tabla 3. Las formaciones discursivas (FD) representan las principales corrientes de significado movilizadas por los agentes centrales (más retuiteados) de los conflictos en análisis. A partir de los datos presentados, es posible identificar la formación de un proceso de antagonismo discursivo de disputa de significados sobre los eventos (Penteado et al. 2021) que se articula en torno a la polarización ideológica entre derecha (de minimización de los efectos de la pandemia, contra medidas del confinamiento, defensa del gobierno de Bolsonaro, ataque a las instituciones y crítica a la izquierda) en oposición a los significados movilizados por los grupos contrarios al gobierno (crítica a la postura negacionista del gobierno de Bolsonaro, defensa de medidas de aislamiento social y ataques a las posturas autoritarias del gobierno) que impregna los cinco conflictos analizados. También es posible identificar el uso de humor en relación con los conflictos, como elemento enunciativo común de la cultura de Internet, que fueron clasificados como indefinidos en este esquema.

Tabla 3. Temas y posicionamiento ideológico

\begin{tabular}{|l|l|l|l|}
\hline \multirow{2}{*}{$\begin{array}{l}\text { Formaciones } \\
\text { Discursivas }\end{array}$} & \multicolumn{3}{|c|}{ Posicionamiento ideológico } \\
\cline { 2 - 4 } & Derecha & Izquierda & Indefinido \\
\hline
\end{tabular}




\begin{tabular}{|c|c|c|c|}
\hline \multirow{2}{*}{$\begin{array}{l}\text { Tema } 1 \\
\text { Salud pública } \\
\text { / pandemia }\end{array}$} & $\begin{array}{l}\text { Minimización de los } \\
\text { efectos de la pandemia }\end{array}$ & $\begin{array}{l}\text { críticas a postura } \\
\text { negacionista de Bolsonaro }\end{array}$ & \multirow[t]{2}{*}{$\begin{array}{l}\text { Humor sobre Covid } \\
\text { y aislamiento social }\end{array}$} \\
\hline & $\begin{array}{l}\text { En contra de las } \\
\text { medidas de aislamiento } \\
\text { social }\end{array}$ & $\begin{array}{l}\text { Defensa del aislamiento } \\
\text { social }\end{array}$ & \\
\hline $\begin{array}{l}\text { Tema } 2 \\
\text { Economía/ } \\
\text { pandemia }\end{array}$ & $\begin{array}{l}\text { En contra medidas de } \\
\text { cierre de empresas } \\
\text { (lockdown) }\end{array}$ & $\begin{array}{l}\text { Falta de preocupación de } \\
\text { Bolsonaro con las víctimas } \\
\text { de Covid }\end{array}$ & $\begin{array}{l}\text { Humor sobre el } \\
\text { confinamiento y } \\
\text { cierre de empresas } \\
\text { por causa da } \\
\text { pandemia }\end{array}$ \\
\hline \multirow{2}{*}{$\begin{array}{l}\text { Tema } 3 \\
\text { Gobierno } \\
\text { federal/ } \\
\text { política }\end{array}$} & $\begin{array}{l}\text { Crítica a la actuación } \\
\text { de la Justifica brasileña } \\
\text { (STF) }\end{array}$ & $\begin{array}{l}\text { Petición de investigación } \\
\text { contra el General Heleno } \\
\text { (ministro militar) }\end{array}$ & \multirow[t]{2}{*}{$\begin{array}{l}\text { Humor sobre la } \\
\text { intervención de los } \\
\text { militares }\end{array}$} \\
\hline & $\begin{array}{l}\text { Apoyo a las } \\
\text { declaraciones del } \\
\text { General Heleno }\end{array}$ & $\begin{array}{l}\text { Acusación de amenaza de } \\
\text { intervención militar del } \\
\text { gobierno de Bolsonaro }\end{array}$ & \\
\hline \multirow{2}{*}{$\begin{array}{l}\text { Tema } 4 \\
\text { Manifestacion } \\
\text { es }\end{array}$} & $\begin{array}{l}\text { Crítica al movimiento } \\
\text { antifascista (violentos e } \\
\text { hipócritas) }\end{array}$ & $\begin{array}{l}\text { Asociación de Bolsonaro } \\
\text { con el fascismo }\end{array}$ & \\
\hline & $\begin{array}{l}\text { Asociación del } \\
\text { movimiento } \\
\text { antifascista con el } \\
\text { terrorismo }\end{array}$ & $\begin{array}{l}\text { Denuncia de persecución al } \\
\text { movimiento antifascista }\end{array}$ & \\
\hline \multirow{2}{*}{$\begin{array}{l}\text { Tema } 5 \\
\text { Educación }\end{array}$} & $\begin{array}{l}\text { Defensa de la medida } \\
\text { de intervención en las } \\
\text { universidades públicas }\end{array}$ & $\begin{array}{l}\text { Defensa de la autonomía } \\
\text { universitaria }\end{array}$ & \multirow[t]{2}{*}{$\begin{array}{l}\text { Humor sobre el uso } \\
\text { de drogas en las } \\
\text { universidades } \\
\text { públicas }\end{array}$} \\
\hline & $\begin{array}{l}\text { Limpieza de la } \\
\text { universidad de la } \\
\text { ideología de izquierda }\end{array}$ & $\begin{array}{l}\text { Gobierno autoritario } \\
\text { (intervenciones en las } \\
\text { universidades) }\end{array}$ & \\
\hline
\end{tabular}

El análisis de los 100 perfiles con más retuits muestra que - aunque algunas cuentas aparecen como polarizadores en más de una red- la mayoría polariza una única red en cada uno de los conflictos analizados. Por esta razón, las 100 cuentas polarizadoras de cada una de las cinco redes suman 433 cuentas y no 500.

Atendiendo al tipo de cuenta, de ellas, tres cuentas aparecen en cuatro redes, dos de ellas se incluyen dentro de la tipología de medios alternativos: RenovaMídia (alineado a la Derecha) y Brasil247 (a la izquierda). La otra es de un político, el diputado Marcelo Freixo — diputado federal por el Partido Socialismo y Libertad (PSOL) - . Entre las 
cuentas que se incluyen en tres conflictos, predominan también las cuentas institucionales o las cuentas de políticos. Por tanto, ese tipo de cuentas son las más influyentes en más de un tema. Sin embargo, juntas apenas representan 68 de las 500 cuentas polarizadoras, lo que supone poco más del 10\%. Es decir, en relación con el $90 \%$ de los casos, el influenciador está ligado directamente a uno de los dos temas. En relación con la alineación ideológica, las cuentas con mención directa a la izquierda representan el 40,8\% del total (204). Las relacionadas con la derecha, suponen el 23,4\% (117), el mismo dato porcentual de cuentas que no permiten identificación con uno de los polos ideológicos. Hay otras 62 cuentas más, clasificadas como «Otras» que constaban como bloqueadas, canceladas, suspendidas o no existentes en el momento de la recogida de datos (el 12,4\% del total de polarizadores).

En relación con la segunda dimensión, la mayor parte de los perfiles influenciadores son cuentas personales (el 74,4\%, un total de 372). Las cuentas institucionales representan el $12,2 \%$ del total (61), un número menor que las clasificadas como «Otras», que son el 13,4\% (67) en el total válido.

Al observar los dos grupos se perciben algunas diferencias. En el caso de cuentas institucionales hay un 47,5\% (29) de cuentas que proceden de medios alternativos, seguida del 24,6\% (15) de cuentas de grupos políticos, el 21,3\% (13) de medios tradicionales y el 6,6\% (4) de otras institucionales. Otros usuarios, que son cuentas personales con entre un mil y un millón de seguidores, representan el 34,8\% (174) del total de cuentas polarizadoras. En segundo lugar, se encuentran las cuentas de políticos en el cargo, siendo un 15\% (75) de los polarizadores personales; seguido de usuarios comunes, que representan el 12,6\% (63); de periodistas, con 8,8\% (44) de polarizadores. Las celebridades e influencers representan, respectivamente, un 1,6\% (8) del total.

Realizadas las descripciones de las categorías, se aborda ahora la presentación de las asociaciones entre los posicionamientos ideológicos y tipos de cuentas, atendiendo a los conflictos o temas analizados en los que estaban presentes:

La tabla 3 muestra la asociación entre el posicionamiento ideológico de los polarizadores y los temas y los porcentuales de distribución de los casos entre las categorías de las dos variables. El coeficiente de asociación V de Cramer muestra una asociación del 25,8\%, aunque estadísticamente significativa, es una asociación que se considera baja. La red «Coronavirus» (tema 1) presenta concentración de posicionamiento indefinido. La red «cnpj» (tema 2) muestra una concentración a la posición ideológica de la izquierda. Las redes «general Heleno» (tema 3 ) y «fascismo» (tema 4) se dividen entre derecha e izquierda. La red «Universidades» (tema 5) muestra concentración de polarizadores próximos a la derecha.

Si se observa el gráfico de correlación de los residuos estandarizados que acompañan a la tabla 3, se aprecia el grado de presencia o ausencia de casos en cada par de categorías, considerando una distribución aleatoria como punto de partida. Así, valores positivos por encima de $+1,96$ son pares de categorías que presentan un número de casos por encima de lo esperado; y los valores negativos por debajo de -1,96 muestra un número de casos inferior al esperado si la distribución fuese aleatoria. Si el valor se aproxima a cero, entonces el número de casos observados se considera próximo a los parámetros esperados y no se considera un valor significativo estadísticamente.

Tabla 4. Posicionamiento ideológico de los polarizadores según temas

\begin{tabular}{l|c}
\hline & Tema \\
\hline
\end{tabular}




\begin{tabular}{|c|c|c|c|c|c|c|}
\hline $\begin{array}{l}\text { Posicionamiento } \\
\text { ideológico }\end{array}$ & $\begin{array}{c}\text { Tema } 1 \\
\text { coronavirus }\end{array}$ & $\begin{array}{l}\text { Tema } 2 \\
\text { Cnpj }\end{array}$ & $\begin{array}{c}\text { Tema } 3 \\
\text { gal_heleno }\end{array}$ & $\begin{array}{c}\text { Tema } 4 \\
\text { fascismo }\end{array}$ & $\begin{array}{c}\text { Tema } 5 \\
\text { universidades }\end{array}$ & Total \\
\hline \multirow[t]{2}{*}{ Indefinido } & 47 & 26 & 19 & 14 & 11 & 117 \\
\hline & $47,0 \%$ & $26,0 \%$ & $19,0 \%$ & $14,0 \%$ & $11,0 \%$ & $23,4 \%$ \\
\hline \multirow[t]{2}{*}{ Derecha } & 8 & 8 & 39 & 38 & 24 & 117 \\
\hline & $8,0 \%$ & $8,0 \%$ & $39,0 \%$ & $38,0 \%$ & $24,0 \%$ & $23,4 \%$ \\
\hline \multirow[t]{2}{*}{ Otro } & 20 & 9 & 7 & 10 & 16 & 62 \\
\hline & $20,0 \%$ & $9,0 \%$ & $7,0 \%$ & $10,0 \%$ & $16,0 \%$ & $12,4 \%$ \\
\hline \multirow[t]{2}{*}{ Izquierda } & 25 & 57 & 35 & 38 & 49 & 204 \\
\hline & $25,0 \%$ & $57,0 \%$ & $35,0 \%$ & $38,0 \%$ & $49,0 \%$ & $40,8 \%$ \\
\hline \multirow[t]{2}{*}{ Total } & 100 & 100 & 100 & 100 & 100 & 500 \\
\hline & $100,0 \%$ & $100,0 \%$ & $100,0 \%$ & $100,0 \%$ & $100,0 \%$ & $100,0 \%$ \\
\hline
\end{tabular}




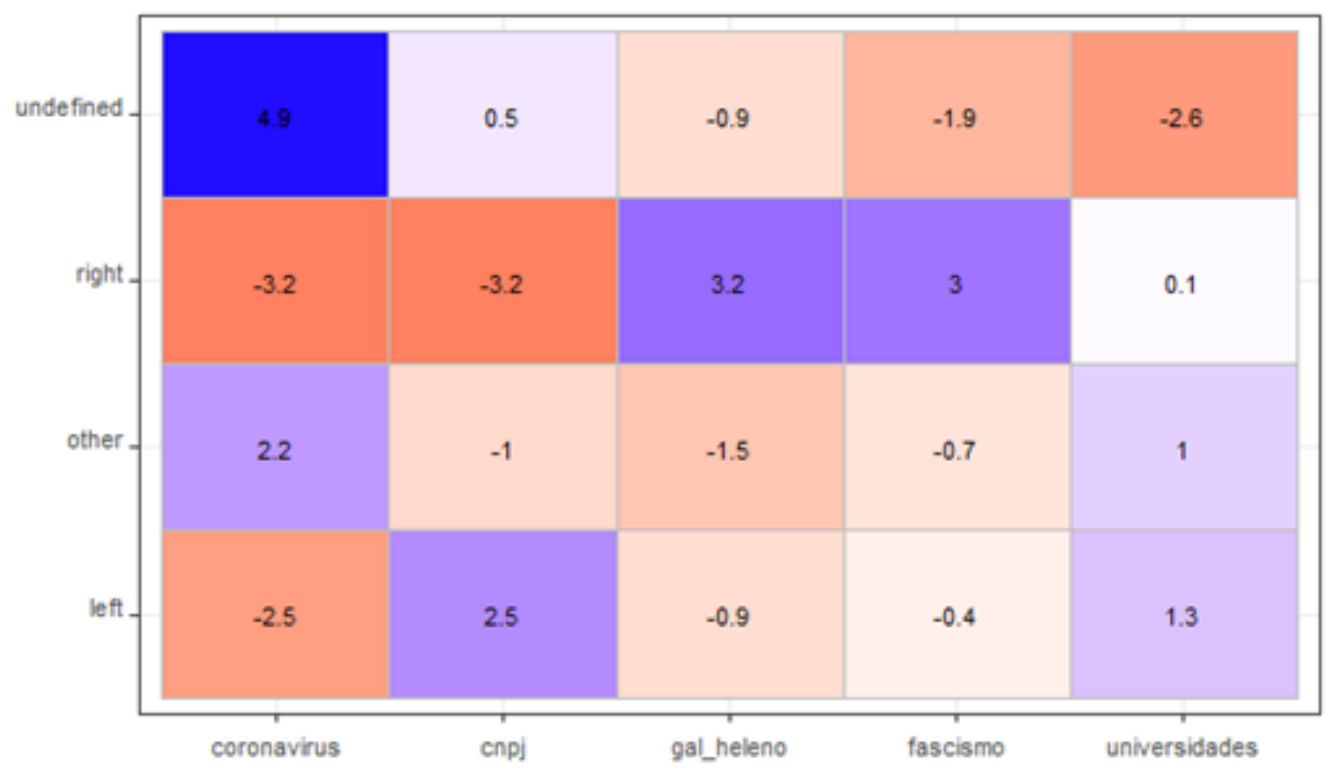

En el caso de las asociaciones positivas entre el tema y el posicionamiento, se aprecia que la categoría dominante es la «Indefinida» asociado al tema 1 (Salud pública / coronavirus). Le sigue la asociación positiva de la categoría «Derecha» para los temas 3 y 4. En el tema 2 - manifestaciones contra el cierre de la economía del país - se encuentran residuos positivos y estadísticamente significativos vinculados con las posiciones de «Izquierda». Y la categoría de «Otros» también se vincula con el primer tema - Salud pública/pandemia—. Todas las demás asociaciones positivas se sitúan por debajo de $+1,96$.

Por lo que se refiere a las asociaciones negativas; es decir, ausencia de casos, las mayores se encuentran entre las categorías «Derecha» y el tema 1 -Salud pública- y el tema 2. La posición «Izquierda» también presenta un alto residuo negativo para el tema 1 - Salud pública/Coronarivus - . Y la posición «Indefinida» muestra residuo negativo con el tema 5 -Universidades-. Todos los demás se sitúan por debajo del límite de 1,96 .

Con relación al tipo de cuenta de los polarizadores y los temas, la tabla 4 muestra los resultados de las distribuciones. En este caso, el coeficiente de asociación está próxima al anterior, con el $22,5 \%$ de asociación, lo que indica una relación baja entre las categorías de las dos variables. Puesto que hay un número mayor de tuiteros, tiende a presentar los mayores porcentuales en las distribuciones, aunque con variaciones. En el caso del tema 1 - Salud pública/Coronarivus - los usuarios comunes y los medios de comunicación tradicionales presentan porcentuales superiores a sus porcentuales totales en las redes. En el caso del tema 2, los tipos de «Usuario común»y «Tuitero» son los de mayores porcentuales relativos. La categoría de «Medios alternativos» y «Periodistas» son los tipos con mayor presencia relativa en la red del tema 3. Por su parte, en el tema 4 se encuentra una distribución de casos muy próxima a la media general de participación en el total. Finalmente, en el último tema, los «Políticos» aparecen con mayor participación proporcional. 
Tabla 5. Categorías de cuentas polarizadoras según temas

\begin{tabular}{|c|c|c|c|c|c|c|}
\hline \multirow[t]{2}{*}{ Categoría } & \multicolumn{5}{|c|}{ Tema } & \multirow[b]{2}{*}{ Total } \\
\hline & $\begin{array}{c}\text { Tema } 1 \\
\text { coronavirus }\end{array}$ & $\begin{array}{c}\text { Tema } 2 \\
\text { Cnpj }\end{array}$ & $\begin{array}{c}\text { Tema } 3 \\
\text { gal_heleno }\end{array}$ & $\begin{array}{c}\text { Tema } 4 \\
\text { fascismo }\end{array}$ & $\begin{array}{c}\text { Tema } 5 \\
\text { universidades }\end{array}$ & \\
\hline \multirow{2}{*}{$\begin{array}{l}\text { Medios } \\
\text { alternativos }\end{array}$} & 7 & 1 & 9 & 6 & 6 & 29 \\
\hline & $8,6 \%$ & $1,1 \%$ & $9,7 \%$ & $6,7 \%$ & $7,1 \%$ & $6,6 \%$ \\
\hline \multirow[t]{2}{*}{ Celebridades } & 2 & 2 & 2 & 2 & 0 & 8 \\
\hline & $2,5 \%$ & $2,2 \%$ & $2,2 \%$ & $2,2 \%$ & $0,0 \%$ & $1,8 \%$ \\
\hline \multirow[t]{2}{*}{ Usuarios comunes } & 16 & 18 & 5 & 11 & 13 & 63 \\
\hline & $19,7 \%$ & $19,8 \%$ & $5,4 \%$ & $12,2 \%$ & $15,5 \%$ & $14,4 \%$ \\
\hline \multirow[t]{2}{*}{ Influencer } & 3 & 1 & 1 & 3 & 0 & 8 \\
\hline & $3,7 \%$ & $1,1 \%$ & $1,1 \%$ & $3,3 \%$ & $0,0 \%$ & $1,8 \%$ \\
\hline \multirow[t]{2}{*}{ Institución } & 1 & 1 & 1 & 0 & 1 & 4 \\
\hline & $1,2 \%$ & $1,1 \%$ & $1,1 \%$ & $0,0 \%$ & $1,2 \%$ & $0,9 \%$ \\
\hline \multirow[t]{2}{*}{ Periodista } & 8 & 8 & 17 & 8 & 3 & 44 \\
\hline & $9,9 \%$ & $8,8 \%$ & $18,3 \%$ & $8,9 \%$ & $3,6 \%$ & $10,0 \%$ \\
\hline \multirow[t]{2}{*}{ Otro } & 1 & 1 & 1 & 1 & 2 & 6 \\
\hline & $1,2 \%$ & $1,1 \%$ & $1,1 \%$ & $1,1 \%$ & $2,4 \%$ & $1,4 \%$ \\
\hline \multirow[t]{2}{*}{ Grupo político } & 1 & 1 & 5 & 5 & 3 & 15 \\
\hline & $1,2 \%$ & $1,1 \%$ & $5,4 \%$ & $5,6 \%$ & $3,6 \%$ & $3,4 \%$ \\
\hline
\end{tabular}




\begin{tabular}{l|r|r|r|r|r|r}
\hline Político & 3 & 11 & 21 & 12 & 28 & 75 \\
\cline { 2 - 7 } & $3,7 \%$ & $12,1 \%$ & $22,6 \%$ & $13,3 \%$ & $33,3 \%$ & $17,1 \%$ \\
\hline $\begin{array}{l}\text { Medios } \\
\text { tradicionales }\end{array}$ & $8,9 \%$ & $0,0 \%$ & $3,2 \%$ & $1,1 \%$ & $1,2 \%$ & $3,0 \%$ \\
\cline { 2 - 7 } & 31 & 47 & 28 & 41 & 27 & 134 \\
\hline Tuitero & $38,3 \%$ & $51,6 \%$ & $30,1 \%$ & $45,6 \%$ & $32,1 \%$ & $39,6 \%$ \\
\cline { 2 - 7 } & 81 & 91 & 93 & 90 & 84 & 439 \\
\hline \multirow{2}{*}{ Total } & $100,0 \%$ & $100,0 \%$ & $100,0 \%$ & $100,0 \%$ & $100,0 \%$ & $100,0 \%$ \\
\cline { 2 - 7 } & & & & & & \\
\hline
\end{tabular}

$\mathrm{V}$ de Cramer $=0,225(\mathrm{p}<0,000)$

\begin{tabular}{|c|c|c|c|c|c|}
\hline tweeter _ & -0.2 & 1.8 & -1.5 & 0.9 & -1.1 \\
\hline Tradtional_Media _ & 3.6 & -1.6 & 0.1 & -1 & -0.9 \\
\hline Politician & -2.9 & -1.2 & 1.3 & -0.9 & 3.6 \\
\hline Political_Group _. & -1.1 & -1.2 & 1 & 1.1 & 0.1 \\
\hline Other - & -0.1 & -0.2 & -0.2 & -0.2 & 0.8 \\
\hline Journalist & 0 & -0.4 & 2.5 & -0.3 & -1.9 \\
\hline Institution - & 0.3 & 0.2 & 0.2 & -0.9 & 0.3 \\
\hline Influencer - & 1.3 & -0.5 & -0.5 & 1.1 & -1.2 \\
\hline Common_User _. & 1.3 & 1.4 & -2.3 & -0.5 & 0.3 \\
\hline Celebrity - & 0.4 & 0.3 & 0.2 & 0.3 & -1.2 \\
\hline Alternative_Media _ & 0.7 & -2 & 1.2 & 0 & 0.2 \\
\hline
\end{tabular}

El gráfico de correlación permite identificar más fácilmente las concentraciones de casos estadísticamente significativas entre los pares de categorías. En la red del tema 1 - Salud pública/coronavirus - la categoría de «Medios tradicionales» es la única que presenta residuos positivos estadísticamente significativos. Y la categoría «Político» es la que muestra residuo negativo estadísticamente significativo. En el tema 2 no se encuentra residuo positivo por encima del límite crítico, pero sí hay una categoría con residuo negativo estadísticamente significativo: «Medios alternativos». En el tema 3 el residuo positivo se encuentra en «Periodistas» y el negativo en «Usuario común». En el tema 4 no existe concentración de casos. En el tema 5 apenas se encuentra un residuo 
positivo estadísticamente significativo, que indica concentración de «Políticos» como polarizadores de esa red.

Lo descrito anteriormente permite identificar que la variable posicionamiento tiene una asociación un poco mayor con los temas, que el tipo de cuenta. Las cuentas polarizadoras vinculadas con la categoría «Derecha» se concentran en los temas 3 y 4; mientras que las polarizadoras de la categoría «Izquierda» se encuentran en los temas 2 y los indefinidos en el tema 1. De la misma forma, la categoría «Medios tradicionales» polarizó principalmente el tema 1 vinculado al coronavirus; la categoría «Político» en el tema 5 y la categoría «Periodista» en el tema 3.

Finalmente, se identifican los tipos de cuentas en función de su distribución en términos de posicionamiento ideológico explícito. La tabla 5 muestra las distribuciones porcentuales solo para los casos válidos, ya que fueron excluidas las categorías «Otros» de las dos variables, por no considerarse tales. El coeficiente de asociación resultó más alto que los anteriores, con un 37,1\%. La categoría «Izquierda» muestra porcentuales por encima del total para el tipo de «Político»; mientras que la categoría «Derecha» refleja porcentuales por encima de los totales para «Medios alternativos» y «Tuitero». La categoría indefinida tiene a vincularse con el «Usuario común», «Periodista» y «Medios tradicionales».

\section{ideológico}

Tabla 6. Distribuciones porcentuales por categoría y posicionamiento

\begin{tabular}{|c|c|c|c|c|}
\hline \multirow[b]{2}{*}{ Tipo } & \multicolumn{4}{|c|}{ Posicionamiento } \\
\hline & Izquierda & Derecha & Indefinido & Total \\
\hline \multirow[t]{2}{*}{ Medio alternativo } & 12 & 12 & 5 & 29 \\
\hline & $5,9 \%$ & $10,6 \%$ & $4,3 \%$ & $6,7 \%$ \\
\hline \multirow[t]{2}{*}{ Celebridad } & 3 & 2 & 3 & 8 \\
\hline & $1,5 \%$ & $1,8 \%$ & $2,6 \%$ & $1,9 \%$ \\
\hline \multirow[t]{2}{*}{ Usuario común } & 29 & 9 & 25 & 63 \\
\hline & $14,2 \%$ & $8,0 \%$ & $21,7 \%$ & $14,6 \%$ \\
\hline \multirow[t]{2}{*}{ Influencer } & 3 & 0 & 5 & 8 \\
\hline & $1,5 \%$ & $0,0 \%$ & $4,3 \%$ & $1,9 \%$ \\
\hline
\end{tabular}




\begin{tabular}{|c|c|c|c|c|}
\hline \multirow[t]{2}{*}{ Institución } & 0 & 0 & 3 & 3 \\
\hline & $0,0 \%$ & $0,0 \%$ & $2,6 \%$ & $0,7 \%$ \\
\hline \multirow[t]{2}{*}{ Periodista } & 10 & 10 & 24 & 44 \\
\hline & $4,9 \%$ & $8,8 \%$ & $20,9 \%$ & $10,2 \%$ \\
\hline \multirow[t]{2}{*}{ Grupo político } & 12 & 2 & 1 & 15 \\
\hline & $5,9 \%$ & $1,8 \%$ & $0,9 \%$ & $3,5 \%$ \\
\hline \multirow[t]{2}{*}{ Político } & 53 & 20 & 2 & 75 \\
\hline & $26,0 \%$ & $17,7 \%$ & $1,7 \%$ & $17,4 \%$ \\
\hline \multirow[t]{2}{*}{ Medio tradicional } & 0 & 0 & 13 & 13 \\
\hline & $0,0 \%$ & $0,0 \%$ & $11,3 \%$ & $3,0 \%$ \\
\hline \multirow[t]{2}{*}{ Tuitero } & 82 & 58 & 34 & 174 \\
\hline & $40,2 \%$ & $51,3 \%$ & $29,6 \%$ & $40,3 \%$ \\
\hline \multirow[t]{2}{*}{ Total } & 204 & 113 & 115 & 432 \\
\hline & $100,0 \%$ & $100,0 \%$ & $100,0 \%$ & $100,0 \%$ \\
\hline
\end{tabular}




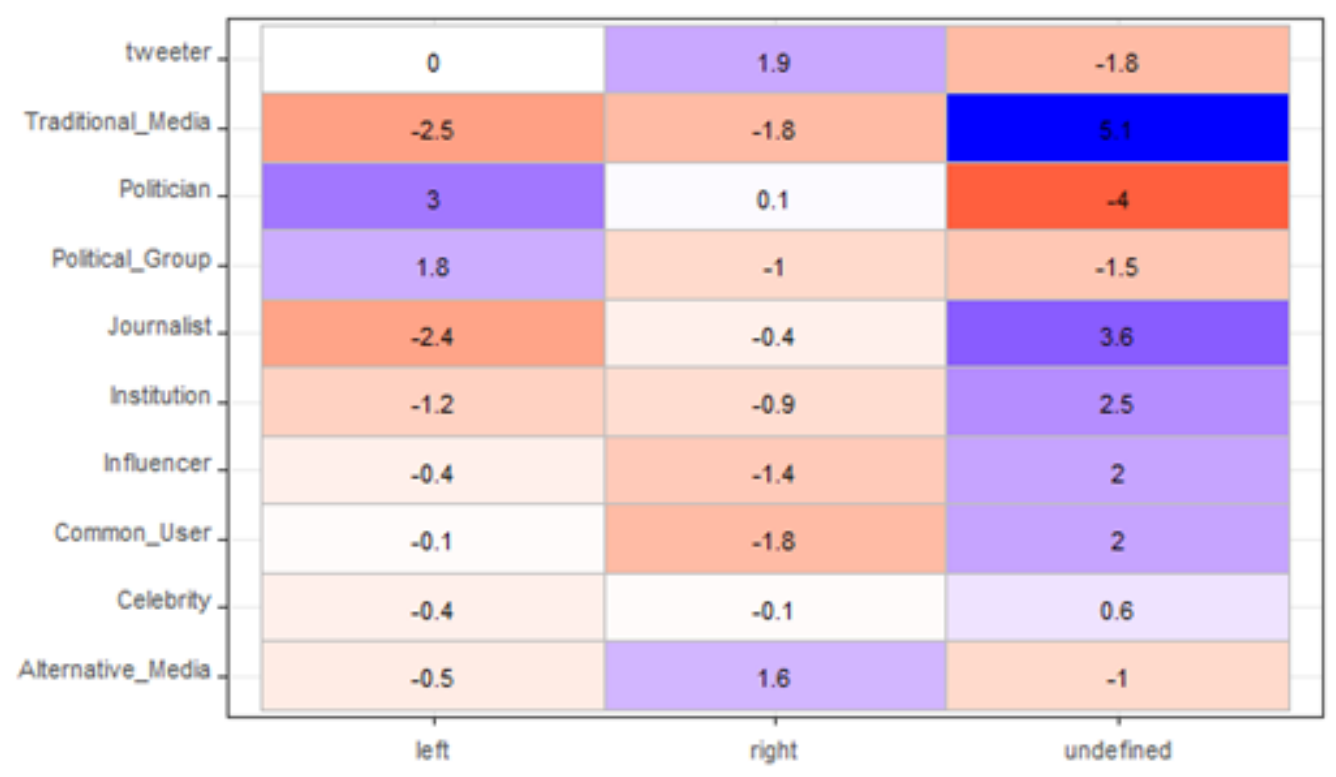

El gráfico de correlación anterior muestra con mayor claridad las asociaciones de presencias y ausencias. En la categoría de «Izquierda» aparece como positiva la categoría «Político» y como negativas, «Medios tradicionales» y «Periodistas». En la categoría «Derecha», hay una distribución más próxima a los límites críticos tanto para la presencia como para la ausencia. La mayor presencia está en «Tuitero», seguida de «Medio alternativo». Las mayores ausencias están en «Medios tradicionales» y «Usuario común». Entre las cuentas con posicionamiento ideológico indefinido, las mayores concentraciones están en «Medios tradicionales», «Periodistas», «Institución», «Usuario común» e «Influencer». El mayor valor negativo para la categoría indefinido está en «Político».

\section{CONCLUSIONES}

Aunque los datos atañen únicamente a un estudio de caso concreto - vinculado con el análisis de las cuentas de usuarios de Twitter en cinco conflictos en Brasil- los datos aportan valor para un análisis más amplio. El contexto político y social brasileño plantea elevados niveles de confrontación y polarización política con proyección internacional. En este contexto, este estudio aborda un análisis de cuentas (500) que se vinculan con cinco temas que generaron conflicto en el país, y tuvieron su manifestación en el debate en Twitter, que supone una de las redes sociales más usadas en ese país; por tanto, puede considerarse representativo del debate político online. Aunque los resultados deben entenderse precisamente en el marco de referencia del país analizado considerando un sistema político presidencialista, diferente por ejemplo al español— sí plantea algunas reflexiones que pueden considerarse extrapolables en algunos casos, por ejemplo:

Los resultados permiten señalar que la esfera pública digital es dinámica, pues en ninguna de las cuentas estuvo entre las más retuiteadas en los cinco temas analizados. Este dato demuestra que, en cada conflicto, dependiendo de la temática, se encuentran nuevos usuarios influyendo en el debate.

Otro dato que llama la atención en esta nueva configuración de la esfera pública digital es que las cuentas personales — como pueden ser la de «Tuitero», «Político», «Usuario común», «Periodistas», «Celebridades» e «Influencers»— son más importantes 
que los perfiles institucionales o colectivos, que representan un 74,4\% de las cuentas identificadas. De estos perfiles de cuentas individuales, destacan los perfiles de «Tuitero» (el 34,8\%) y de «Político» (el 15\%) como los más retuiteados, que utilizan sus redes personales para la difusión de contenidos. Esto muestra la personalización y customización del debate público, también con la entrada de nuevos actores o fuentes en la discusión política, en el caso de «Tuitero». Vale destacar, igualmente, que los «Medios tradicionales» pierde espacio como fuente de información en el debate público, ya que únicamente 13 perfiles (el 2,6\% de perfiles de esta categoría aparecen entre los mensajes más retuiteados, seguido de «Usuarios comunes» $(12,6 \%)$, «Periodistas» $(8,8 \%)$, «Medios alternativos» $(4,2 \%)$ y «Grupos políticos» $(3 \%)$. Esta configuración favorece la polarización política y la desinformación.

Los datos también señalan la politización y la polarización en los diferentes conflictos analizados. Entre los principales hallazgos, encontramos que, dependiendo del tema, la red presenta un tipo específico de usuarios con mayor número de retuits. En los temas más distantes de política partidaria — como el tema 1, coronavirus - la posición ideológica de los usuarios más activos es indefinida y ellos son predominantemente pertenecientes a los medios tradicionales y a los periodistas.

Los usuarios más intensos con un perfil más próximo a la categoría «Derecha» tendieron a estar más presentes en los temas ligados a aspectos de gobierno, como el tema 3 , o de carácter ideológico, como el tema 4. Se trata de una clara apropiación del tema por parte de los simpatizantes del gobierno de Bolsonaro, en este caso. En el tema 5, los usuarios más retuiteados fueron los políticos y los usuarios comunes vinculados a la categoría «Izquierda», con una clara vinculación y apropiación de la red por parte de la oposición del gobierno federal.

De esa forma, el análisis realizado aquí apunta a que los usuarios más retuiteados escogen las batallas que quieren lidiar. Y esa selección, aunque no intencionada y desorganizada, parece ser colectiva. Así, entre las asociaciones de posicionamiento ideológico y tipo de cuenta se encuentra literatura en esta línea. Los usuarios vinculados a la categoría «Derecha» tienden a estar entre los «Tuitero» y «Medios alternativos», originarios de las redes sociales online. Mientras que los usuarios vinculados a la categoría «Izquierda» están principalmente entre los «Políticos», y los usuarios con posición ideológica indefinida se vinculan con medios tradicionales y periodistas.

\section{REFERENCIAS BIBLIOGRÁFICAS}

Barberá, Pablo, y Zeitzoff, Thomas. 2018. «The new public address system: why do world leaders adopt social media?». International Studies Quarterly, 62(1): 121-130.

Bimber, Bruce y Gil de Zúñiga, Homero. 2020. «The unedited public sphere». New Media \& Society, 22(4): 700-715.

Bruns, Axl. 2019. Are filter bubbles real? John Wiley \& Sons.

Carvalho, Cássia de França, Fabrício, Goya, Denise., \& de Camargo Penteado, Claudio 2016. «Brazilians divided: Political protests as told by twitter». En Transactions on Large-Scale Data-and Knowledge-Centered Systems XXVII, ed. Anjomshoaa, Amin, Hung, Patrick, Kalisch, Dominik, Sobolevsky, Stanislav (pp. 1-18). Springer: Berlin, Heidelberg. https://doi.org/10.1007/978-3-662-53416-8_1

Casero-Ripollés, Andreu, Feenstra, Ramón y Tormey, Simon. 2016. «Old and new media logics in an electoral campaign: The case of Podemos and the two-way street mediatization of politics». The international journal of press/politics, v. 21, n. 3 : 378-397. https://doi.org/10.1177/1940161216645340

Casero-Ripollés, Andreu. 2018. «Research on political information and social media: Key 
points and challenges for the future». El profesional de la información, v. 27, n. 5: 964-974.

Castells, Manuel. 2015. Networks of outrage and hope: Social movements in the Internet age. Cambridge; Polity Press.

Chadwick, Andrew. 2017. The hybrid media system: Politics and power. New York: Oxford University Press.

Chaves-Montero, Alfonso, Relinque-Medina, Fernando, Fernández-Borrero, Manuela. Á., Vázquez-Aguado, Octavio. 2021. «Twitter, Social Services and Covid-19: Analysis of Interactions between Political Parties and Citizens». Sustainability, Vol.13: 1-15, 2187. https://doi.org/10.3390/su13042187

Dahlberg, Lincoln. 2007. «Rethinking the fragmentation of the cyberpublic: from consensus to contestation». New Media \& Society, Vol. 9 No. 5: 827-847.

De Blasio, Emiliana., Kneuer, Mariane, Schünemann, Wolf y Sorice, Michele. 2020. «The Ongoing Transformation of the Digital Public Sphere: Basic Considerations on a Moving Target». Media and Communication, 8(4), 1-5.

De Blasio, Emiliana y Sorice, Michele. 2018. «Populism between direct democracy and the technological myth». Palgrave Commun, 4, 15: 1-11.

Dufva, Tommi y Dufva, Mikko. 2019. «Grasping the future of the digital society». Futures, 107: 17-28.

Freelon, Deen, McIlwain, Charlton, y Clark, Meredith. 2018. «Quantifying the power and consequences of social media protest». New Media \& Society, 20(3): 990-1011.

García Marzá, Domingo. 1999. Teoria de la democràcia. Colección Universitas, 3. Castelló de la Plana: Publicacions de la Universitat Jaume I.

Gerbaudo, Paolo. 2012. Tweets and the streets: Social media and contemporary activism. London: Pluto Press.

Gerbaudo, Paolo. 2018. «Social media and populism: an elective affinity?». Media, Culture \& Society, 40(5): 745-753.

Gualda, Estrella y Rúas, José. 2019. «Conspiracy theories, credibility and trust in information». Communication \& Society, 32(1), 179-194.

Igual Castelló, Cristina. 2017. «Solimàn el Magnifico y Roxolona. El poder del turco en la cultura visual y escrita de Occidente». Potestas, 9: 233-260.

Laclau, Ernesto y Mouffe, Chantal. 2014. Hegemony and socialist strategy: Towards a radical democratic politics. London: Verso Trade.

López Cantos, Francisco José. 2016a. Tecnología audiovisual. Castelló de la Plana: Publicacions de la Universitat Jaume I.

López Cantos, Francisco José. 2016b. Tecnología de la comunicación. Castelló de la Plana: Publicacions de la Universitat Jaume I.

López-García, Guillermo 2017. «Comunicación política y discursos sobre el poder». El profesional de la información, v. 26, n. 4: 573-578

Luengo, Óscar y Fernández-García, Belén. 2019. «Campaign Coverage in Spain: Populism, Emerging Parties, and Personalization». In Mediated Campaigns and Populism in Europe, eds. Nombre y apellidos de editores (pp. 99-121). Lisboa: Palgrave Macmillan, Cham.

Montlleó Peris, Rosa, Iván Medall Peris y Alfredo Fornas Pallarés. 2014. Biografies rescatades del silenci. Experiències de guerra i posguerra a Castelló. Castelló de la Plana: Publicacions de la Universitat Jaume I.

Moro Ipola, Micaela y Laura Mezquita Guillamón. 2015. Entrena't les neurones: programa de rehabilitació neurocognitiva per a pacients amb trastorn mental greu. Castelló de la Plana: Publicacions de la Universitat Jaume I.

Narvaz, Martha Giudice, Nardi, Henrique Caetano, y Morales, Blanca. 2006. «Nas tramas 
do discurso: A abordagem discursiva de Michel Pêcheux e de Michel Foucault». Revista de Psicologia Política, 6(12): 369-390.

Primario, Simoneta; Borrelli, Dario; Iandoli, Luca; Zolo, Giuseppe y Lipizzi, Carlo. 2017. «Measuring polarization in twitter enabled in online political conversation: The case of 2016 us presidential election». En 2017 IEEE International Conference on Information Reuse and Integration (IRI), 607-613. San Diego: IEEE.

Penteado, Claudio Luis de Camargo, Goya, Denise, H. y França, Fabrício Olivetti de. 2021. «Discursive conflicts around the impeachment of Dilma Rousseff (Brazil) on Twitter». In Perspetivas da Contemporaneidade, 1(1): 49-67.

Penteado, Claudio Luis de Camargo, Chaves, Julia y Marks, Santana. 2021. «O discurso anti PT como estratégia política: Estudo da campanha eleitoral de Jair Bolsonaro no Twitter nas eleições de 2018». En Impactos político-comunicacionais nas eleições brasileiras de 2018, v. 1, orgs. Emerson Cervi, Maria Elena Weber (pp. 87-111). Curitiba: CPOP / Carvalho comunicação.

Recuero, Raquel, Zago, Gabriela, Bastos, Marco y Araújo, Ricardo. 2015. «Hashtags functions in the protests across Brazil». Sage Open, 5(2): 1-14.

Serrano-Contreras, Ignacio-Jesús, García-Marín, Javier, y Luengo, Óstermcar. 2020. «Measuring online political dialogue: does polarization trigger more deliberation?». Media and Communication, 8(4): 63-72.

Soares, Felipe, Recuero, Raquel., y Zago, Gabriela. 2019. «Asymmetric polarization on Twitter and the 2018 Brazilian presidential elections». En Proceedings of the 10th international conference on social media and society (pp. 67-76New York: Association for Computing Machinery (ACM).

Souza, Rafael Martins, Graça, Luís Felipe y Silva, Ralf. 2017. «Politics on the web: using Twitter to estimate the ideological positions of Brazilian representatives». Brazilian Political Science Review, 11(3): 1-26.

Strömbäck, Jesper, y Luengo, Óscar G. 2008. «Polarized pluralist and democratic corporatist models: A comparison of election news coverage in Spain and Sweden». International Communication Gazette, 70(6): 547-562.

Stromer-Galley, Jennifer. 2019. Presidential campaigning in the Internet age. New York: Oxford University Press.

van Dijck, Jose, Poell, Thomas y de Waal, Martijn. 2018. Platform society: Public values in a connective world. Oxford: Oxford University Press.

Welch, Kathleen E. 1999. Electric Rhetoric: Classical Rhetoric, Oralism and a New Literacy. Cambridge: MIT Press. http://netlibrary.com.

Woolley, Samuel y Howard, Philip. 2017. Computational propaganda worldwide: Executive summary.

Wolton, Dominique. 2000. Internet ¿y después? Barcelona: Gedisa. 